\title{
Quality of Life After Surgery for Colon Cancer in Patients With Lynch Syndrome: Partial Versus Subtotal Colectomy
}

\author{
Jasmijn F. Haanstra, M.D. ${ }^{1}$ - Wouter H. de Vos tot Nederveen Cappel, M.D., Ph.D. ${ }^{1}$ \\ Jessica P. Gopie, M.Sc. ${ }^{2}$ 'Juda Vecht, M.D., Ph.D. ${ }^{1}$ Steven A. L. W. Vanhoutvin, M.Sc. ${ }^{3}$ \\ Annemieke Cats, M.D., Ph.D. ${ }^{3}$ Hester J. van der Zaag-Loonen, M.D., Ph.D. ${ }^{4}$ \\ Alexandra M. J. Langers, M.D., Ph.D. ${ }^{5}$ Jerry H. W. Bergmann, M.D. ${ }^{6}$ \\ Paul C. van de Meeberg, M.D., Ph.D. ${ }^{7}$ Evelien Dekker, M.D., Ph.D. ${ }^{8}$ \\ Jan H. Kleibeuker, M.D., Ph.D. ${ }^{9}$ • Hans F. A. Vasen, M.D., Ph.D. ${ }^{10}$ \\ Fokko M. Nagengast, M.D., Ph.D. ${ }^{11}$ Peter van Duijvendijk, M.D., Ph.D. ${ }^{12}$ \\ 1 Department of Gastroenterology and Hepatology, Isala Clinics, Zwolle, Netherlands \\ 2 Department of Clinical Genetics, Leiden University Medical Center, Leiden, Netherlands \\ 3 Department of Gastroenterology and Hepatology, The Netherlands Cancer Institute Antoni van Leeuwenhoek Hospital, \\ Amsterdam, Netherlands \\ 4 Department of Epidemiology, Gelre Hospitals, Apeldoorn, Netherlands \\ 5 Department of Gastroenterology and Hepatology, Leiden University Medical Center, Leiden, Netherlands \\ 6 Department of Gastroenterology and Hepatology, Martini Hospital, Groningen, Netherlands \\ 7 Department of Gastroenterology and Hepatology, Slingeland Hospital, Doetinchem, Netherlands \\ 8 Department of Gastroenterology and Hepatology, Academic Medical Center, Amsterdam, Netherlands \\ 9 Department of Gastroenterology and Hepatology, University Medical Center Groningen, University of Groningen, \\ Groningen, Netherlands \\ 10 Netherlands Foundation for the Detection of Hereditary Tumors, Leiden, Netherlands \\ 11 Department of Gastroenterology and Hepatology, Radboud University Medical Center Nijmegen, Nijmegen, Netherlands \\ 12 Department of Surgery, Radboud University Medical Center Nijmegen, Nijmegen, Netherlands, and Gelre Hospitals, \\ Apeldoorn, Netherlands
}

BACKGROUND: Lynch syndrome is a disorder caused by mismatch repair gene mutations. Mutation carriers have a high risk of developing colorectal cancer. In patients with Lynch syndrome in whom colon cancer has been diagnosed, in general, subtotal colectomy instead of partial colectomy is recommended because of the substantial risk of metachronous colorectal cancer. However, the effect of more extensive surgery on quality of life and functional outcome is unknown.

Disclosures: None reported.

Poster presentation at the meeting of InSiGHT, San Antonio, TX, March 30 to April 2, 2011 and at Digestive Disease Week, Chicago, IL, May 7 to 10,2011. Presented at the meeting of the Netherlands Society of Gastroenterology, Veldhoven, the Netherlands, March 22 to 23, 2011.

Correspondence: Jasmijn F. Haanstra, M.D., Department of Gastroenterology and Hepatology, University Medical Center Groningen, PO Box 30001, 9700 RB Groningen, the Netherlands. E-mail jf.haanstra@umcg.nl

Dis Colon Rectum 2012; 55: 653-659

DOI: 10.1097/DCR.0b013e31824f5392

(C)The ASCRS 2012

Diseases of the Colon \& Rectum Volume 55: 6 (2012)
OBJECTIVE: The aim of this study was to investigate quality of life and functional outcome in patients with Lynch syndrome after partial colectomy and subtotal colectomy.

DESIGN: This is a nationwide cross-sectional study in the Netherlands.

SETTINGS: Two quality-of-life questionnaires (Short Form-36 and The European Organization for Research and Treatment of Cancer Colorectal Cancer-specific Quality of Life Questionnaire Module) and a functional outcome questionnaire (Colorectal Functional Outcome) were used.

PATIENTS: Patients with Lynch syndrome who underwent surgery for colon cancer were included.

MAIN OUTCOME MEASURES: The primary outcomes measured were quality of life and functional outcome.

RESULTS: Questionnaires were sent to 192 patients with Lynch syndrome who underwent surgery for colorectal cancer. A total of 136 patients returned the questionnaire (response rate, $71 \%$ ). Eighteen patients with rectal cancer, 9 patients with a permanent ileostomy, and 5 
patients with an IPAA were excluded. Fifty-one patients underwent partial colectomy, and 53 underwent subtotal colectomy. None of the scales of the Short Form-36 survey showed a significant difference. Analysis of the Colorectal Functional Outcome questionnaire revealed that, after subtotal colectomy, patients have a significantly higher stool frequency $(p \leq 0.01)$ and a significantly higher score on stool-related aspects $(p=0.06)$ and social impact $(p=0.03)$. The European Organization for Research and Treatment of Cancer Colorectal Cancerspecific Quality of Life Questionnaire Module presented more problems with defecation after subtotal colectomy $(\mathrm{p} \leq 0.01)$.

LIMITATIONS: Certain selection bias cannot be ruled out. CONCLUSIONS: Although functional outcome is worse after subtotal colectomy than after partial colectomy, generic quality of life does not differ after the 2 types of surgery in Lynch syndrome. When discussing the options for surgery with the patient, all advantages and disadvantages of both surgical procedures, including quality of life and functional outcome, should be discussed.

KEY WORDS: Lynch syndrome; Quality of life; Functional outcome; Colon cancer; Surgery.

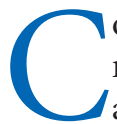
olorectal carcinoma (CRC) is one of the most common cancers worldwide. ${ }^{1,2}$ In approximately $5 \%$ of all cases, CRC is associated with a highly penetrant dominant or recessive inherited syndrome. The most common of these is Lynch syndrome (hereditary nonpolyposis colorectal cancer). ${ }^{3}$ Lynch syndrome (LS) is an autosomal dominant disorder caused by a mutation in one of the mismatch repair genes. ${ }^{4,5}$ Mutation carriers have a lifetime risk of approximately $25 \%$ to $75 \%$ to develop CRC. In addition, they have an increased risk of developing endometrial cancer and various other malignancies. ${ }^{4-6}$

Because of the high risk of developing CRC, gene mutation carriers are advised to participate in intensive colonoscopic surveillance programs. The aim of these programs is to detect and remove adenomatous polyps, the premalignant lesions of CRC, and to detect cancers at an early stage. Most authors recommend subtotal colectomy when CRC is diagnosed, especially in young patients because of the high risk of a metachronous CRC after partial colectomy. Several studies reported that the risk of developing metachronous CRC varies between 22\% and $41 \% .^{7-9}$ We found a 10 -year cumulative risk of developing CRC after partial colectomy of $16 \%$ in comparison with $3 \%$ after subtotal colectomy. ${ }^{10}$ Moreover, with the use of a mathematical (Markov) model, we observed that subtotal colectomy was associated with a slightly increased life expectancy. ${ }^{11}$ In this study, the overall life expectancy gain of subtotal colectomy in comparison with hemicolectomy at ages 27,47 , and 67 was $2.3,1$, and 0.3 years, respectively. When CRC is diagnosed above the age of 60 years, partial colectomy appears to be justified, because the expected survival benefit is small. ${ }^{11,12}$

To date, there are no studies comparing quality of life (QoL) and functional outcome after both types of surgery in patients with LS. The question whether QoL differs between partial and subtotal colectomy is becoming more important because of the recently reported low risk of developing CRC while under surveillance. ${ }^{13}$ If a substantial difference in QoL would exist, the recommendation for subtotal colectomy in young patients with CRC might be revised.

In addition, for adequate patient education, information regarding functional outcome and QoL is essential. The aim of the present study, therefore, was to investigate QoL and functional outcome after partial colectomy and subtotal colectomy in patients with LS.

\section{METHODS}

\section{Study Design}

This study was a nationwide cross-sectional study in the Netherlands to investigate whether patients with LS who underwent a subtotal colectomy have a different QoL and functional outcome in comparison with patients that underwent partial colectomy.

\section{Study Population}

The study population consisted of 288 patients with LS living in the Netherlands who underwent a partial or subtotal colectomy for CRC and were registered at the Dutch Lynch Syndrome Registry.

Patients were considered to have LS if they were proven, obligate, or probably gene carriers. Untested persons from LS families who developed CRC under the age of 50 were defined as probably gene carriers. Patients were excluded if they underwent surgery less than 1 year before March 15, 2010, the starting date of the study. Metastatic disease was also an exclusion criterion. The study was approved by the University Medical Center St. Radboud Nijmegen Medical Ethical Committee.

\section{Procedure}

Patients with LS registered at the Dutch Lynch Syndrome Registry who underwent a colectomy for CRC were identified. This national registry for families with hereditary CRC was established in the Netherlands in 1987. The registry had 3 objectives: 1) to promote surveillance in LS families; 2) to guarantee the continuity of the surveillance program; and 3) to promote research in this field. The methods and approach of the registry have been described elsewhere. ${ }^{14}$ Subsequently, the specialists were asked for 
permission to contact their patients. When their specialist approved, patients were sent 3 validated questionnaires concerning QoL and functional outcome. From all respondents, patients with rectal cancer, a permanent ileostomy, or an IPAA were excluded, because these types of surgery are very different in comparison with colon surgery alone. The remaining respondents were subsequently classified into 2 groups based on the type of surgery they had undergone. The first group consisted of patients who had undergone a partial colectomy. Partial colectomy was defined as a segmental resection, ileocecal resection, right or left hemicolectomy, transversectomy, or sigmoid resection. The second group included patients that underwent a subtotal colectomy. Patients were thought to have undergone a subtotal colectomy if the surgeon had documented the procedure as such, or if at least the total colon, with the exception of the rectum and, in many patients, also a part of the sigmoid, was resected. Patients who had more than one resection belonged to the partial colectomy group when the level of the anastomosis was above the sigmoid and if more than $50 \mathrm{~cm}$ of colorectum was still in situ.

\section{Instruments}

Three questionnaires were sent to the patients. To investigate generic QoL, the Short Form-36 (SF-36) health survey was sent. To assess disease-specific QoL, the European Organization for Research and Treatment of Cancer Colorectal Cancer-specific Quality of Life Questionnaire Module (EORTC QLQ CR-38) was sent. To obtain information about bowel function, the Colorectal Functional Outcome (COREFO) questionnaire was used.

SF-36. The SF-36 is one of the most used generic instruments about the QoL. The SF-36 consists of 36 multiple-choice questions. These questions are divided into 8 domains: physical functioning, role limitations due to physical health problems, bodily pain, general health perceptions, vitality, social functioning, role limitations due to emotional problems, and general mental health. The raw data are converted into a scale running from 0 to 100 . A higher score indicates a higher level of functioning or well-being. ${ }^{15,16}$

EORTC QLQ CR-38. EORTC QLQ CR-38 is a specific instrument to measure QoL. It is developed for patients with CRC. This questionnaire consists of 38 multiplechoice questions. Nineteen questions are answered by subgroups: namely by female or male patients or by patients with or without a stoma. The EORTC QLQ CR-38 consists of 2 function domains (body image and sexual function) and 7 symptom domains (micturition problems, GI tract symptoms, chemotherapy side effects, defecation problems, stoma-related problems, and male and female sexual problems). Moreover, 3 single items are part of the questionnaire: sexual enjoyment, future perspective, and weight loss. The raw scores are summed into domains and rescaled from 0 to 100. A higher score indicates better functioning for all functioning scales and for the single items, sexual enjoyment and future perspective. A higher score at the symptom scales and the single item weight loss corresponds with a higher level of symptomatology. ${ }^{17,18}$

COREFO. The COREFO questionnaire is a reliable and valid instrument consisting of 27 multiple-choice questions to evaluate the functional outcome after colorectal surgery. This questionnaire is divided into 5 scales: incontinence, social impact, frequency, defecation-related aspects, and use of drugs. The data are converted into a score of 0 to 100 ; a higher score indicates a worse functional outcome. ${ }^{19}$

\section{Statistical Analysis}

The statistical analysis was performed with SPSS version 16.0. Student $t$ tests were used for group comparisons. Proportions were compared by $\chi^{2}$ tests. The Mann-Whitney $U$ test was used for comparing follow-up. $p$ values less than 0.05 were considered statistically significant. Incomplete questionnaires were handled in accordance with the standards in the user manuals of the questionnaires.

\section{RESULTS}

A total of 288 living patients with LS who had undergone a colectomy because of CRC were identified at the Dutch Lynch Syndrome Registry. Six of them died shortly before the start of the survey. For 86 patients, the specialist did not respond to our invitation letter or did not allow us to approach their patients. Three patients had moved and their new address was unknown by the closing date of the study. One patient was excluded because of metastatic disease. In total, 192 patients were invited. One hundred thirty-six questionnaires were returned. The response rate was $71 \%$. None of these patients underwent surgery less than 1 year before the starting date of the study.

Nonresponders were not different from responders with respect to age, sex, type of surgery, pathological stage of CRC, and type of the mismatch repair mutation. Based on the type of surgery 18 patients with rectal cancer, 9 patients with a permanent ileostomy, and 5 patients with an IPAA were excluded. Fifty-one patients had undergone a partial colectomy and 53 patients a subtotal colectomy (Fig. 1). The mean time period between the surgical procedure and time of survey was 11.6 years (range, 1.3-32.3 years). In the subtotal colectomy group, the mean length of colorectum in situ measured at colonoscopy was $23 \mathrm{~cm}$ (range, 5-50 cm). Groups were comparable with respect to most clinical features (Table 1). However, patients in 


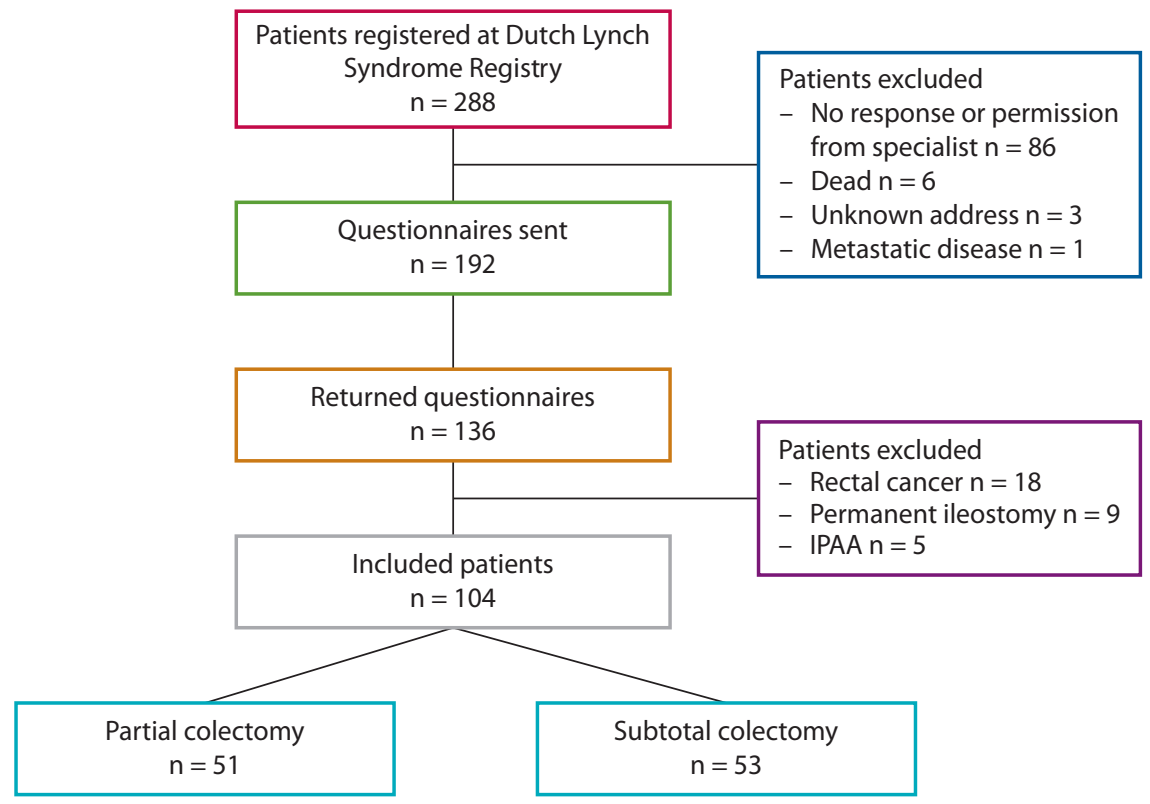

FIGURE 1. Study design.

the partial colectomy group had a significantly longer follow-up.

\section{QoL and Functional Outcome}

The results of the SF-36 are shown in Figure 2. No significant differences were found between both groups with regard to generic QoL, as measured by the SF-36. Figure 3 demonstrates the results of the EORTC QLQ CR-38. Patients in the subtotal colectomy group had a significantly higher score on defecation problems $(p<0.01)$. Patients in the partial colectomy group had a significantly higher score on the "male sexual problems" scale $(p=0.02)$.

Figure 4 shows the results of the COREFO questionnaire. Patients in the subtotal colectomy group reported a significantly higher stool frequency $(p<0.01)$ and had a significantly higher score on the scales social impact $(p=0.03)$ and stool-related aspects $(p=0.06)$.

\section{DISCUSSION}

This cross-sectional study demonstrates that generic QoL does not differ after subtotal colectomy and partial colectomy in patients with LS. After subtotal colectomy, patients have a worse functional outcome in terms of stool frequency, stool-related aspects, and social impact in comparison with patients who underwent a partial colectomy.

To our knowledge, this is the first study that evaluated the QoL and bowel function after colectomy in patients with LS. However, similar studies have been published for other patient populations. You et $\mathrm{al}^{20}$ retrospectively reviewed 321 resections. Both bowel function and QoL after subtotal colectomy were inferior to bowel function and QoL after segmental colectomy. A possible explanation for the different outcome in QoL in comparison with our study might be differences in patient demographics and indication for surgery. The most common indication

\section{TABLE 1. Clinical data}

\begin{tabular}{|c|c|c|c|c|c|}
\hline & \multicolumn{2}{|c|}{$\begin{array}{c}\text { Partial colectomy } \\
(n=51)\end{array}$} & \multicolumn{2}{|c|}{$\begin{array}{l}\text { (Sub)total } \\
\text { colectomy } \\
(n=53)\end{array}$} & \multirow[b]{2}{*}{$p$} \\
\hline & $n$ & $\%$ & $n$ & $\%$ & \\
\hline Sex & & & & & $0.44^{\mathrm{a}}$ \\
\hline Male & 25 & 49 & 30 & 57 & \\
\hline Female & 26 & 51 & 23 & 43 & \\
\hline Mutation type & & & & & $0.38^{\mathrm{a}}$ \\
\hline MLH1 & 23 & 45 & 25 & 47 & \\
\hline $\mathrm{MSH} 2$ & 19 & 37 & 24 & 45 & \\
\hline MSH6 & 8 & 16 & 4 & 8 & \\
\hline Unknown & 1 & 2 & & & \\
\hline Mutation status & & & & & $0.61^{\mathrm{a}}$ \\
\hline Proven gene carrier & 42 & 82 & 41 & 77 & \\
\hline Obligate gene carrier & 3 & 6 & 4 & 8 & \\
\hline Probably gene carrier & 6 & 12 & 8 & 15 & \\
\hline Stadium carcinoma & & & & & $0.11^{\mathrm{a}}$ \\
\hline Dukes A + B & 38 & 75 & 46 & 87 & \\
\hline Dukes C & 13 & 25 & 7 & 13 & \\
\hline More resections & & & & & $0.28^{\mathrm{a}}$ \\
\hline Yes & 5 & 10 & 9 & 17 & \\
\hline No & 46 & 90 & 44 & 83 & \\
\hline Age, y, mean (SD) & 59.0 & 11.9 & 58.0 & 10.0 & $0.64^{b}$ \\
\hline $\begin{array}{l}\text { Follow-up after surgery, } \\
\text { y, median } \\
\text { (IQR 25-75) }\end{array}$ & 12.7 & $8.5-18.7$ & 9.2 & $4.2-12.0$ & $<0.01^{c}$ \\
\hline
\end{tabular}

$\mathrm{IQR}=$ interquartile range. Quartile 25 and 75 .

${ }^{a} \chi^{2}$ test.

${ }^{\mathrm{b}}$ Student $t$ test.

cMann-Whitney $U$ test. 


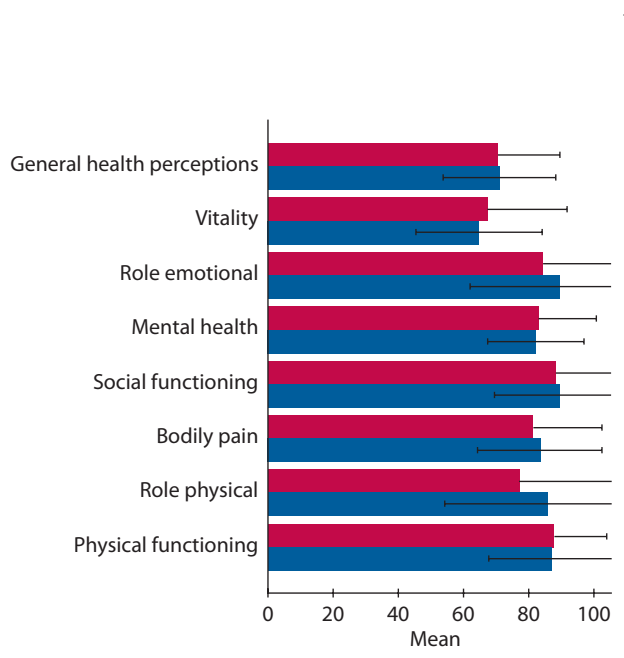

FIGURE 2. Results SF-36. A higher score represents a higher level of functioning. Error bars, \pm 1 SD. SF-36 = Short Form-36 health survey.

for extended resection was multiple polyps, particularly in younger patients with previous resections, whereas segmental resections were primarily performed in older women with CRC. Moreover, possibly not all patients reached the plateau in their functional outcome, because patients were surveyed at various time intervals (including less than 1 year) after surgery. Clinical experience and previous studies suggest that it takes 6 to 12 months for bowel function to stabilize postoperatively. In addition, right-sided colonic resections resulting in ileocolonic anastomosis were excluded from You's study. This surgical procedure is common especially in LS. Because of all these differences, it is difficult to compare these results with our study.

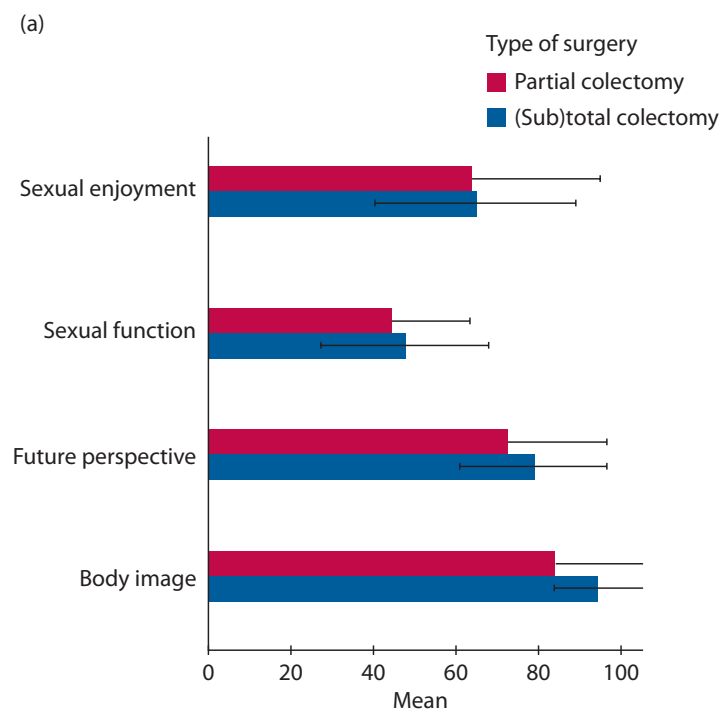

The SCOTIA group prospectively compared subtotal colectomy with segmental resection in patients with malignant left-sided CRC. ${ }^{21}$ No significant difference in general health status was found, which corresponds to our results.

Subtotal colectomy seems to have a worse functional outcome and a higher social impact than partial colectomy, as measured by the COREFO. Although functional outcome is worse after subtotal colectomy, no difference in social functioning and generic QoL as measured by the SF-36 seems to exist after both types of surgery. This suggests that a worse functional outcome cannot be extrapolated to a worse QoL. Ko et $\mathrm{al}^{22}$ also documented that better functional results do not necessarily equate to better QoL in patients with familial adenomatous polyposis.

It is possible that general QoL does not differ between subtotal colectomy and partial colectomy in patients with LS because, after a subtotal colectomy, LS patients are less anxious about developing a metachronous CRC. Also, only regular sigmoidoscopy is needed for surveillance, and patients less often need secondary surgery. Patients also possibly adapt and become accustomed to the new situation and functional outcome after a long follow-up. Another explanation might be that the SF-36 is not sensitive enough to detect subtle differences in QoL determined by minor operative resection extents.

QoL scores of the subscales of the SF-36, in general, were comparable to normal scores of the Dutch national population. However, on the subscale "mental health," patients seemed to score higher in comparison with the Dutch population. ${ }^{15}$ A possible explanation might be that some cancer survivors report posttraumatic growth. A

(b)

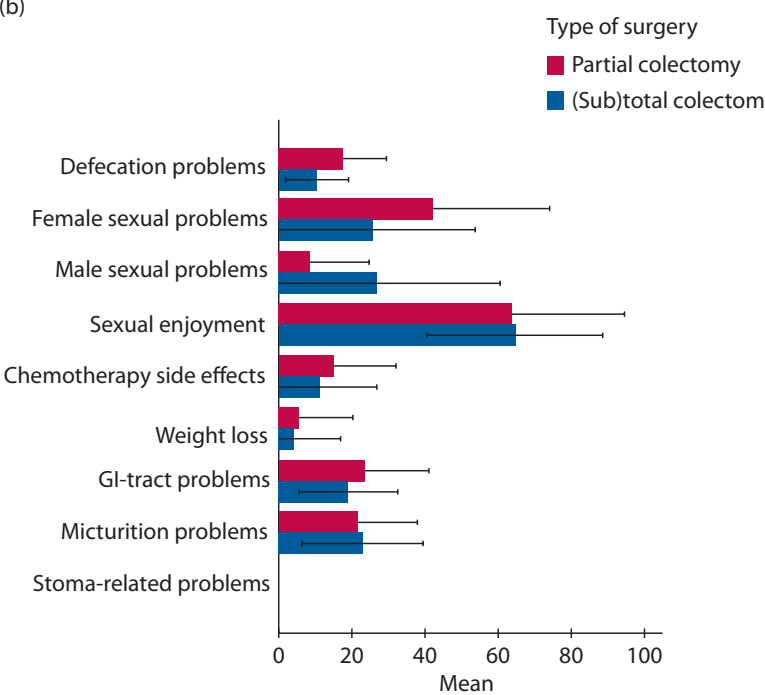

FIGURE 3. Results EORTC QLQ CR-38. A, Functional scales and single items (sexual enjoyment and future perspective): A higher score indicates better functioning. Error bars, \pm 1 SD. B, Symptom scales and the single item weight loss: A higher score indicates a higher level of symptomatology. Error bars, \pm 1 SD. EORTC QLQ CR-38 = European Organization for Research and Treatment of Cancer Colorectal Cancerspecific Quality of Life Questionnaire Module. 


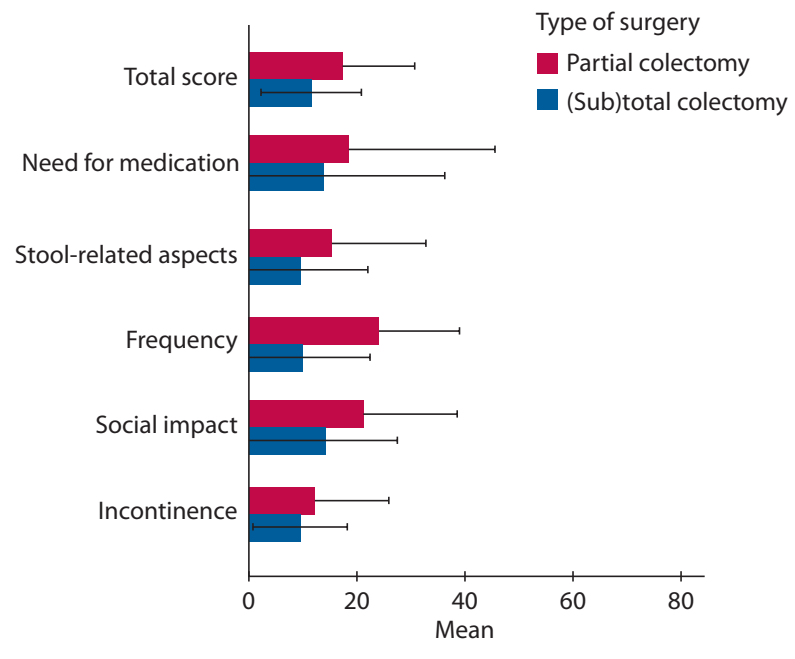

FIGURE 4. Results COREFO. Higher scores represent a higher level of symptomatology. Error bars, $\pm 1 \mathrm{SD}$. COREFO = Colorectal Functional Outcome.

younger age at diagnosis, as is the case in LS, is associated with greater posttraumatic growth. ${ }^{23,24}$

An unexpected finding of this study was that, in comparison with subtotal colectomy, male patients report significantly more sexual problems after partial colectomy. We do not have an explanation for this observation.

Several limitations of the current study should be noted. First, we cannot rule out certain selection bias. The present study was a cross-sectional study to examine QoL in patients with LS after colectomy, which might have lead to selection bias by selective nonresponse. However, responders did not differ from nonresponders in terms of objective criteria, and the response rate was relatively high $(71 \%)$. Hence, the influence of selective nonresponse is probably small. Second, selection bias could have occurred by selection of patients with little comorbidity for subtotal colectomy instead of partial colectomy. However, the influence of this is probably low, because CRC in LS patients generally occurs at a relatively young age. Because we included a relatively large group of patients with similar ages in both groups, we assumed similar comorbidity. However, theoretically, the 2 groups could have differed in comorbidity.

Another limitation might be the classification of people with multiple resections in the partial or subtotal group. However, subanalysis of patients with only 1 resection demonstrated no differences. Ideally, QoL should be evaluated at different time periods. Therefore, we suggest future studies with a prospective design with measurements before and after surgery.

What are the implications of this study concerning the management of screen-detected CRC in LS? Owing to the many factors that should be considered in the decisionmaking process, it is difficult to provide a general recom- mendation for all patients. ${ }^{25}$ Subtotal colectomy reduces the risk of metachronous CRC in comparison with partial colectomy and subsequently leads to a slightly increased life expectancy. Contemporaneously, generic QoL does not differ after the 2 types of surgery. Therefore, we consider subtotal colectomy as the treatment of choice in young patients (eg, <60 years) with CRC detected under surveillance. Obviously, all advantages and disadvantages of both surgical procedures should be discussed with the patient.

\section{CONCLUSION}

Although functional outcome is worse after subtotal colectomy than after partial colectomy, generic QoL does not differ after the 2 types of surgery in patients with LS. Patients should be informed about possible differences in functional outcome.

\section{ACKNOWLEDGMENTS}

The authors thank M. Velthuizen for all the support and help during study progress. We are also indebted to the participants of the project. This study would not have been possible without the efforts of all LS patients included in the study.

\section{REFERENCES}

1. Gellad ZF, Provenzale D. Colorectal cancer: national and international perspective on the burden of disease and public health impact. Gastroenterology. 2010;138:2177-2190.

2. Parkin DM, Bray F, Ferlay J, et al. Global cancer statistics, 2002. CA Cancer J Clin. 2005;55:74-108.

3. Lynch HT, de la Chapelle A. Hereditary colorectal cancer. $N$ Engl J Med. 2003;348:919-932.

4. Quehenberger F, Vasen HF, van Houwelingen HC. Risk of colorectal and endometrial cancer for carriers of mutations of the hMLH1 and hMSH2 gene: correction for ascertainment. J Med Genet. 2005;42:491-496.

5. Vasen HF, Wijnen JT, Menko FH, et al. Cancer risk in families with hereditary nonpolyposis colorectal cancer diagnosed by mutation analysis. Gastroenterology. 1996;110:1020-1027.

6. Koornstra JJ, Mourits MJ, Sijmons RH, et al. Management of extracolonic tumours in patients with Lynch syndrome. Lancet Oncol. 2009;10:400-408.

7. Parry S, Win AK, Parry B, et al. Metachronous colorectal cancer risk for mismatch repair gene mutation carriers: the advantage of more extensive colon surgery. Gut. 2011;60:950-957.

8. Mecklin JP, Järvinen H. Treatment and follow-up strategies in hereditary nonpolyposis colorectal carcinoma. Dis Colon Rectum. 1993;36:927-929.

9. Van Dalen R, Church J, McGannon E, et al. Patterns of surgery in patients belonging to Amsterdam-positive families. Dis Colon Rectum. 2003;46:617-620.

10. de Vos tot Nederveen Cappel WH, Nagengast FM, Griffioen $\mathrm{G}$, et al. Surveillance for hereditary nonpolyposis colorectal cancer: a long-term study on 114 families. Dis Colon Rectum. 2002;45:1588-1594. 
11. de Vos tot Nederveen Cappel WH, Buskens E, van Duijvendijk $\mathrm{P}$, et al. Decision analysis in the surgical treatment of colorectal cancer due to a mismatch repair gene defect. Gut. 2003;52:1752-1755.

12. Maeda T, Cannom RR, Beart RW Jr, et al. Decision model of segmental compared with total abdominal colectomy for colon cancer in hereditary nonpolyposis colorectal cancer. J Clin Oncol. 2010;28:1175-1180.

13. Vasen HF, Abdirahman M, Brohet R, et al. One to 2-year surveillance intervals reduce risk of colorectal cancer in families with Lynch syndrome. Gastroenterology. 2010;138:2300-2306.

14. Vasen HF, den Hartog Jager FC, Menko FH, et al. Screening for hereditary non-polyposis colorectal cancer: a study of 22 kindreds in The Netherlands. Am J Med. 1989;86:278-281.

15. Aaronson NK, Muller M, Cohen PD, et al. Translation, validation, and norming of the Dutch language version of the SF-36 Health Survey in community and chronic disease populations. J Clin Epidemiol. 1998;51:1055-1068.

16. Ware JE Jr., Gandek B. Overview of the SF-36 Health Survey and the International Quality of Life Assessment (IQOLA) Project. J Clin Epidemiol. 1998;51:903-912.

17. Sprangers MA, te Velde A, Aaronson NK. The construction and testing of the EORTC colorectal cancer-specific quality of life questionnaire module (QLQ-CR38). European Organization for Research and Treatment of Cancer Study Group on Quality of Life. Eur J Cancer. 1999;35:238-247.

18. van Duijvendijk P, Slors JF, Taat CW, et al. Quality of life after total colectomy with ileorectal anastomosis or proctocolecto- my and ileal pouch-anal anastomosis for familial adenomatous polyposis. Br J Surg. 2000;87:590-596.

19. Bakx R, Sprangers MA, Oort FJ, et al. Development and validation of a colorectal functional outcome questionnaire. Int $\mathrm{J}$ Colorectal Dis. 2005;20:126-136.

20. You YN, Chua HK, Nelson H, et al. Segmental vs. extended colectomy: measurable differences in morbidity, function, and quality of life. Dis Colon Rectum. 2008;51:1036-1043.

21. The SCOTIA Study Group. Single-stage treatment for malignant left-sided colonic obstruction: a prospective randomized clinical trial comparing subtotal colectomy with segmental resection following intraoperative irrigation: subtotal colectomy versus ontable irrigation and anastomosis. Br J Surg. 1995;82:1622-1627.

22. Ko CY, Rusin LC, Schoetz DJ Jr, et al. Does better functional result equate with better quality of life? Implications for surgical treatment in familial adenomatous polyposis. Dis Colon Rectum. 2000;43:829-835.

23. Barskova T, Oesterreich R. Post-traumatic growth in people living with a serious medical condition and its relations to physical and mental health: a systematic review. Disabil Rehabil. 2009;31:1709-1733.

24. Salsman JM, Segerstrom SC, Brechting EH, et al. Posttraumatic growth and PTSD symptomatology among colorectal cancer survivors: a 3-month longitudinal examination of cognitive processing. Psychooncology. 2009;18:30-41.

25. Vasen HF, de Vos tot Nederveen Cappel WH. Cancer: Lynch syndrome-how should colorectal cancer be managed? Nat Rev Gastroenterol Hepatol. 2011;8:184-186. 